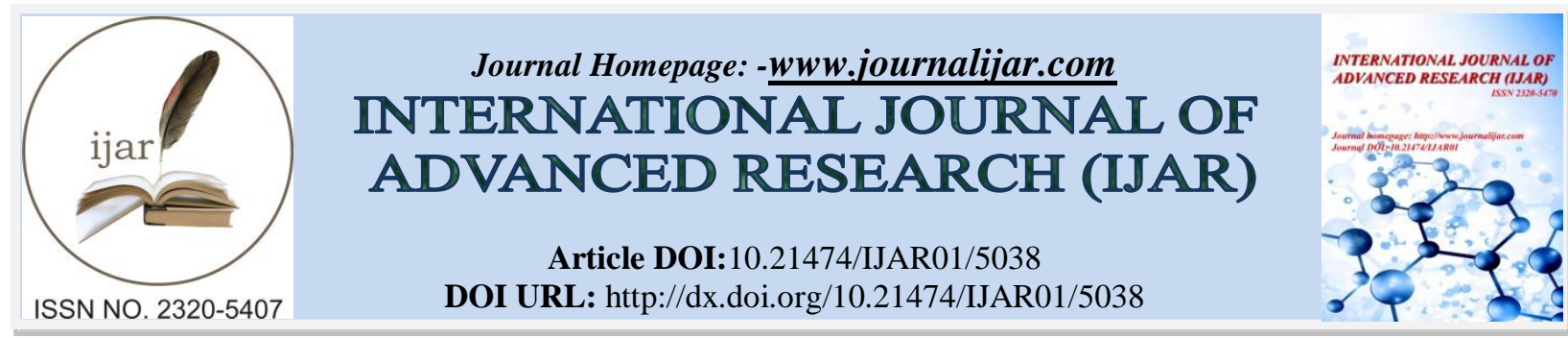

RESEARCH ARTICLE

\title{
ROLE OF BNP AS A SCREENING TOOL TO IDENTIFY ASYMPTOMATIC CARDIAC DISEASE IN TYPE 2 DIABETIC PATIENTS.
}

\author{
Madhusudhanan $\mathbf{P}^{1}$, Narayanan $\mathbf{M}^{2}$, Karthik $\mathrm{S}^{3}$ and Ezhumalai ${ }^{4}$. \\ 1. Junior Resident. \\ 2. Professor. \\ 3. Assistant Professor. \\ 4. Senior Statistician.
}

\section{Manuscript Info}

Manuscript History

Received: 01 June 2017

Final Accepted: 03 July 2017

Published: August 2017

Key words:-

bnp- brain natriuretic peptide, tmt- tread mill test

\section{Abstract}

Background:-Diabetic adults have increased risk of mortality from atheroscelortic heart diseases compared to non diabetic humans. Routinely these patients have a increased new cases of cardiac failure and the unfavourable outcome of diabetes on cardiac failure, might worsening reflect an underlying diabetic cardiomyopathy aggrevated by coronary artery disease and left ventricular hypertrophy unidentified and untreated cases until advanced disease causes disability.this detain could be avoided if investigating techniques could be utilised to identify silent ischemia in its preclinical phase

Aims and objectives:-Bnp as a screening tool to identify silent myocardial ischemia in asymptomatic type 2 diabetic patients. The objective of the study is how bnp value will be useful in early detection of myocardial ischemia without subjecting the patient to tmt.our effort is to identify a simple blood test which is highly sensitive in identifying myocardial ischemia.

Methods:-A total of 100 patients in our out patient department of general medicine with type 2 diabetic patients were taken up for study,their fasting and post prandial blood sugar,fasting lipid profile,renal function test, liver function test,bnp values checked and tmt results were reported.their bnp and tmt reports were analysed and compared statistically.

Results:-Out of 100 diabetic patients 22 patients had ischemia in tmt ,remaining 78 patients had negative results in tmt. The results reveal that bnp, tgl and total cholesterol are better model in detecting asymptomatic cardiac diseaseamong type 2 diabetic patients in complement to tmt method. The cut-off value 760 yield a sensitivity of 100 and false positive rate (1-specificity) 2.6bnp with cut-off value at 760 can be administered in detecting asymptomatic cardiac disease in complement to tmt with sensitivity 100 and false positive rate 2. 6 Conclusion:-This study shows a clear correlation between occurrence of increase in ischemia with increase in nt pro bnp concentration. Furthermore, the results suggest that ntprobnp value above $760 \mathrm{pg} / \mathrm{ml}$ is a good indicator for referring a patient for exercise tolerance test.we conclude that a single measurement of nt pro bnp at the diabetic opd 
can provide important information about the cardiac status of asymptomatic diabetic patients who might require a cardiac evaluation.early diagnosis of cardio vascular complications with the simple blood test would help preventing and reducing mortality and morbidity in diabetic patients

Copy Right, IJAR, 2017,. All rights reserved.

\title{
Introduction:-
}

Diabetec adults have increased risk of mortality from atheroscelortic heart diseases compared to non diabetic humans(1). Routinely these patients have a increased new cases of cardiac failure and the unfavourable outcome of diabetes on cardiac failure,might worsening reflect an underlying diabetic cardiomyopathy aggrevated by coronary artery disease and left ventricular hypertrophy. Large number patients with myocardial ischemia continue to exist as unidentified and untreated cases until advanced disease causes disability.this detain could be avoided if invetigating techniques could be utilised to identify silent ischemia in its preclinical phase.

Analysis of serum brain natriuretic peptide (bnp) is a helpful bio marker of myocardial ischemia, and bnp values are in higher side in patients with coronary artery disease(2).we sought to regulate the total cases of cad,lvh,, and subclinical lv dysfunction in diabetic patients without known cardiac disease and whether a uncomplicated technique on the basis of serum bnp measurements could be utilised to evaluate patients likely to have cad ,lvh, and abnormal myocardial function.

Almost fifty percent of cardiac deaths occurs in people without prior history of cardiac diseases(3). Sudden cardiac deaths more common in diabetic patients who have a higher risk of coronary artery disease that is often silent.to avoid such events the first step would be to develop a simple test that can identify those diabetic patients with silent coronary artery disease.recent studies have led us to hypothesizes that b-type natriuretic peptide may be able to identify silent ischemia.

Our hypothesis is based on three main observations.first goetze et al showed that ischemic myocardial tissues express more brain natriuretic peptide than normal cardiac tissues(4).second cardiomyocytes degranulate and release bnp when they are made to be hypoxic(5).third bnp can identify the presence of cad and myocardial ischemia in symptomatic angina patients.(6)

Our objective of the study is how bnp value will be useful in early detection of myocardial ischemia without subjecting the patient to tmt.

Our effort is to identify a simple blood test which is highly sensitive in identifying myocardial ischemia. Since bnp is secreted from myocardial cell fibres even in any subclinical lvd,bnp may be found increased levels in blood.

\section{Aims and objectives:-}

Aim:-bnp as screening tool to identify silent myocardial ischemia in asymptomatic type 2 diabetic patients

\section{Objectives:-}

The objective of the study is how bnp value will be useful in early detection of myocardial ischemia without subjecting the patient to tmt.our effort is to identify a simple blood test which is highly sensitive in identifying myocardial ischemia

\section{Materials and methods:-}

\section{Study subjects:-}

The study involves 100 diabetic patients fulfilling inclusion criteria attending out patient department of general medicine clinic.

\author{
Type of study:- \\ Cross sectional study:- \\ Place of study:- \\ Department of general medicine, mahathma gandhi medical college\& research institute.
}




\section{Selection process:-}

Study population:-

This study will be conducted in a minimum of 100 diabetic patients fulfilling inclusion criteria attending out patient department of general medicine clinic.

\section{Volunteers recruitment process:-}

No volunteers are included in this study.

\section{Inclusion criteria:-}

1. Male and female patients of age $>30$ years were selected.

2. Subjects who are diabetic for $>3$ years with or without treatment were included.

3. Blood pressure -110-140/70-90 mmhg with or without treatment.

4. Subjects with no history of heart disease or complaints related to cardiac disease like history of cad, moderate to severe valvular disease, atrial fibrillation, or other severe arrhythmias and congenital heart disease were enrolled in to the study.

5. Subject with no history of end stage renal failure.

\section{Exclusion criteria:-}

1. Subjects with presence of pathological q waves, lv hypertrophy on voltage criteria, or st/t wave abnormalities in ecg.

2. History of congestive heart failure.

3. History of end stage renal failure.

4. History of smoking

5. History of alcohol

6. Subject with uncontrolled hypertension

7. Any abnormal $\mathrm{rft}, \mathrm{lft}$ reports on screening

8. Patient who is not willing to participate in the study

9. Individuals who are cognitively impaired and/or who are unable to give informed consent.

10. Any other health or mental condition that in the investigator's opinion may adversely affect the subject's ability to complete the study.

\section{Sampling procedure:-}

Not applicable. All consecutive patients fulfilling the selection criteria are included in the study.

No of groups:- One.

\section{Sample size:-}

The sample size of my study is 100

\section{Results:-}

Out of 100 diabetic patients 22 patients had ischemia in tmt ,remaining 78 patients had negative results in tmt. 


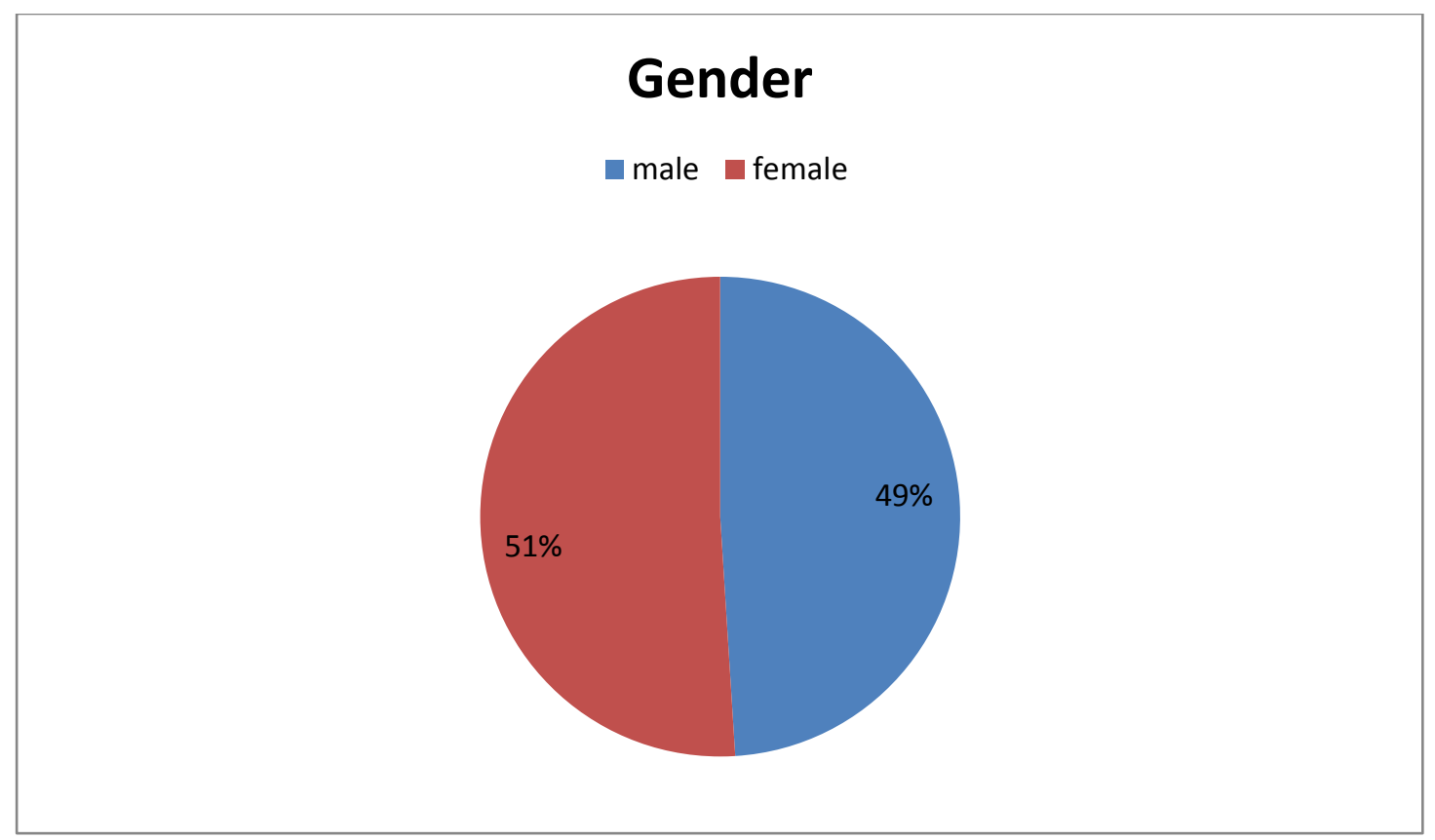

Fig 1:-Gender distribution of our study

\begin{tabular}{|l|l|}
\hline \multicolumn{2}{|l|}{ Case processing summary } \\
\hline Tmt & Valid $\mathrm{n}$ (listwise) \\
\hline Positive $\mathrm{a}^{\mathrm{a}}$ & 22 \\
\hline Negative & 78 \\
\hline
\end{tabular}

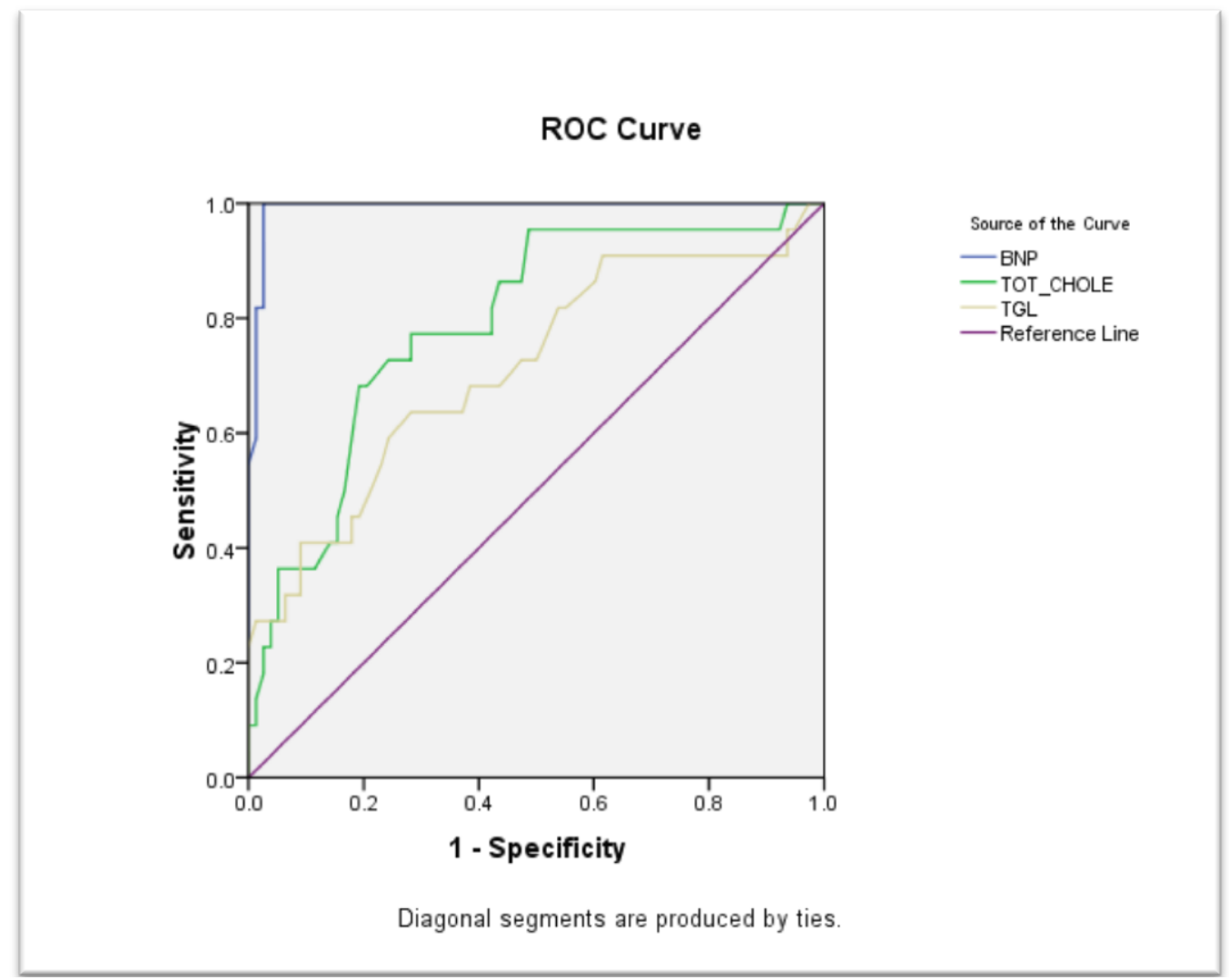

Fig 2:-Roc showed comparision between sensitivity and specificity based on bnp,total cholesterol,triglycerides 
An roc curve represents the tradeoff between sensitivity and false positive rate (1-specificity) of a test in identifying the true cases. An roc curve was drawn to compare the sensitivity and false positive (1-specificity) in identifying asymptomatic cardiac disease using bnp, tgl and total cholesterol. The result shows that the presence of asymptomatic cardiac disease was 22 and absence was 78 based on tmt. The result shows that the area under the curve for bnp is 0.992 with standard error 0.007 (95\% ci, 0.979 to 1.000). The auc for total cholesterol was 0.791 with standard error 0.054 (95\% ci, 0.684 to 0.897 ). The auc for tgl was 0.713 with standard error 0.067 (95\% ci, 0.582 to 0.844$)$. The results reveal that bnp, $\operatorname{tgl}$ and total cholesterol are better model in detecting asymptomatic cardiac diseaseamong $\mathrm{t} 2$ diabetic patients in complement to tmt method. However, the graph shows that bnp is the best among the other model in developing a cut off value to detect asymptomatic cardiac disease among $\mathrm{t} 2 \mathrm{diabetic}$ patients.

Hence, the coordinates for the curve for sensitivity and false positive rate was drawn for bnp in detecting asymptomatic cardiac disease. Table shows that the cut-off value for bnp in detecting all positive cases as +ve was 100 at 233 to 760 and reduces thereof. However, false positive rate (1-specificity) ie., reporting negative cases as positive for the same cut-off value ranging from 100 to 2.6. Ie the cut-off value 760 yield a sensitivity of 100 and false positive rate (1-specificity) 2.6. On the other hand, when the bnp cut-off value was increased to 796.5, the sensitivity reduced to 90.9 but false positive rate was unaltered with 2.6. In order to get a higher sensitivity rate and low false positive rate, the cut-off vale for bnp is fixes at 760 . Hence, it may be concluded that bnp with cut-off value at 760 can be administered in detecting asymptomatic cardiac disease in complement to tmt with sensitivity 100 and false positive rate 2.6

\section{Discussion:-}

Commonly tmt,echo, and bnp have been clinically used for screening for and monitoring ischemic heart disease and lv dysfunction in patients who have high possibility of development of cad and lv dysfunction.the present study has demonstrated that asymptomatic diabetic subjects may identify significant numbers of patients with asymptomatic ischemia.measuring bnp is simple and rapid and has been suggested test for myocardial ischemia in patients with diabetic mellitus, bnp levels in the present study were significantly different between patients with and without myocardial ischemia.

\section{Screening for cad:-}

Coronary artery disease is commonly silent in diabetic patients, and screening with stress echocardiography may be of value in identifying patients with occult cad.however, although the cardiac natriuretic peptide system is involved in the pathophysiology of intimal plaque formation in human beings and plasma bnp concentration has been shown to be markedly increased in patients with coronary artery disease,even with out concomitant lv dysfunction,the present study suggests that bnp levels can be used for cad screening.our findings showing that bnp is elevated in silent ischemic heart disease in type 2 diabetic patients.

\section{Bnp in diabetes:-}

Most importantly, the balance of evidence suggests that bnp is not influenced by diabetes itself- bnp release has no relation with acute hyper insulinemic conditions, diabetes does not influence in the expression of bnp messenger in the ventricular myocardium.bnp does not increase in response to acute hyperglycemia in diabetic patients.the association of type 2 diabetes and obesity may be important ,as obesity is associated with low bnp.overall,these data suggest that diabetes have no effect on bnp synthesis.

Since the study population group is very small it can not be genaralized for entire population.hence more studies are required in larger groups to identify whether bnp can be used as a screening tool to identify asymptomatic cardiac disease among diabetic patients at primary health care centre level the current cost of bnp is little high,when we use it in a bigger scale we hope that cost might get reduced in future.

\section{Conclusion:-}

This study shows a clear correlation between occurrence of increase in ischemia with increase in nt pro bnp concentration. Furthermore, the results suggest that ntprobnp value above $760 \mathrm{pg} / \mathrm{ml}$ is a good indicator for referring a patient for exercise tolerance test.we conclude that a single measurement of nt pro bnp at the diabetic opd can provide important information about the cardiac status of asymptomatic diabetic patients who might require a 
cardiac evaluation.early diagnosis of cardio vascular complications with the simple blood test would help preventing and reducing mortality and morbidity in diabetic patients

\section{References:-}

1. self-reported heart disease and stroke among adults with and without diabetes-united states, 1999-2001. Mmwr morb mortal wkly rep 2003;52:1065-70.

2. Goetze jp, christoffersen c, perko $\mathrm{m}$, et al. Increased cardiac bnp expression associated with myocardial ischemia. Faseb j 2003; 17:1105 -7.

3. Vreede-swagemakers jj, gorgels ap, dubois-arbouw wi, et al. Out-ofhospital cardiac arrest in the 1990's: a population-based study in the maastricht area on incidence, characteristics and survival. $\mathrm{J}$ am coll cardiol 1997;30:1500-5

4. Goetze jp, christoffersen c, perko m, et al. Increased cardiac bnp expression associated with myocardial ischemia. Faseb j 2003; 17:1105-7.

5. Hopkins we, chen $\mathrm{z}$, fukagawa nk, et al. Increased atrial and brain natriuretic peptides in adults with cyanotic congenital heart disease: enhanced understanding of the relationship between hypoxia and natriuretic peptide secretion. Circulation 2004;109:2872-7.

6. Weber $\mathrm{m}$, dill $\mathrm{t}$, arnold $\mathrm{r}$, et al. $\mathrm{N}$-terminal b-type natriuretic peptide predicts extent of coronary artery disease and ischemia in patients with stable angina pectoris. Am heart j 2004;148:612-20. 